\title{
A Study on Speckle Noise Removal and Segmentation of Retinal Layers in OCT Image Analysis
}

\author{
Priyanka Kaushik \\ Department of ECE \\ Gauhati University Institute of Science and \\ Technology, Gauhati University
}

\author{
S. R. Nirmala, $\mathrm{PhD}$ \\ Department of ECE \\ Gauhati University Institute of Science and \\ Technology, Gauhati University
}

\begin{abstract}
The survey paper shows the application of Optical Coherence Tomography images for detection of retinopathy. Image analysis methods enormously help in distinguishing different eye ailments. Currently, determination of retinal diseases depends mostly upon optical imaging techniques. Optical coherence tomography (OCT) is a routine diagnostic imaging method used worldwide in the evaluation of retinal diseases using the cross-sectional view of the retinal layers. The primary challenge in automatic identification and analysis of retinal disease cases is the presence of speckle noise and variation across edge boundaries. Due to the complexity of retinal structures, the tediousness of manual segmentation and variation from different specialists, many methods have been proposed to aid with this analysis. Therefore, efforts are being made to improve clinical decision making based on automated analysis of OCT data which will result in improving the accuracy, precision, and computational speed of segmentation methods, as well as reducing the amount of manual interaction.
\end{abstract}

\section{Keywords}

Optical Coherence Tomography, Retinal Layers, Image denoising, Image Segmentation, Macular Edema

\section{INTRODUCTION}

Retinopathy is any harm to the retina of the eyes, which may cause vision hindrance. Retinopathy refers to retinal vascular illness, or harm to the retina caused by abnormal blood flow. Age-related Macular Degeneration (AMD) is an illness that causes weakening visual impairment in a huge number of people around the world. Diabetic Macular Edema (DME) is a main reason for vision loss in patients with diabetes. Glaucoma is a multi-factorial optic neuropathy described by dynamic basic loss of Retinal Ganglion cells (RGC) that may bring about vision loss and irreversible visual impairment. Retinopathy of Prematurity (ROP) is a disorder of advancement of retinal veins in premature babies[1].

Optical Coherence Tomography (OCT) is an indicative imaging instrument that gives cross sectional image of the retina. It is utilized to decide the degree and measure of retinal thickening. It was to begin with depicted in 1991 by Huang and his colleagues, turned out to be commercially accessible in 1996. OCT works by projecting a broadband light into the eye, with the most common commercial systems revolved around $840 \mathrm{~nm}$ wavelength. The backscattered light is then combined with light reflected from a reference arm for comparison. The combination of both lights generates an interference signal. As the beam of light sweeps across the retina, these interference patterns are handled to shape the cross-sectional images of the living tissue [2].
In order to study retinal OCT images, the layers of the retina must be known. This is shown in the figures below. The retina can be divided into 10 layers including [3]:

- $\quad$ The Inner Limiting Membrane (ILM).

- The Nerve Fiber Layer (NFL).

- The Ganglion Cell Layer (GCL).

- $\quad$ The Inner Plexiform Layer (IPL).

- The Inner Nuclear Layer (INL).

- $\quad$ The Outer Plexiform Layer (OPL).

- The Outer Nuclear Layer (ONL).

- The Outer Limiting Membrane (OLM).

- The Photoreceptor Layer (PL).

- The Retinal Pigmented Epithelium (RPE).

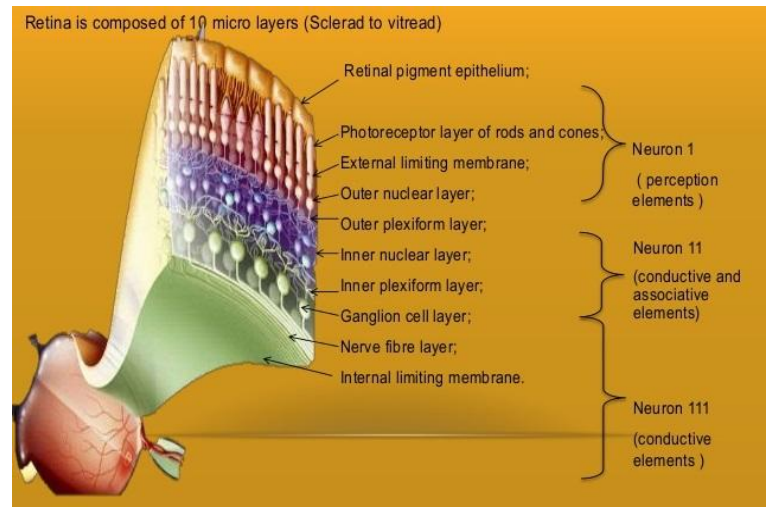

Figure 1: Different Retinal Layers (Image Courtesy: https://www.slideshare.net/Lhacha/retina-and-layers)

Since optical coherence tomography (OCT) gives crosssection images of retina, ophthalmologists can see each of the retina's distinctive layers. This allows ophthalmologist to map and measure their thickness. These measurements help with early diagnosis.

\section{RELATED LITERATURE}

This section provides a study on the previous works carried out by researchers in the field of OCT. Here papers on noise removal and segmentation have been discussed. First three papers on noise removal are discussed and then five papers on segmentation and lastly two papers on macular edema have been discussed. 


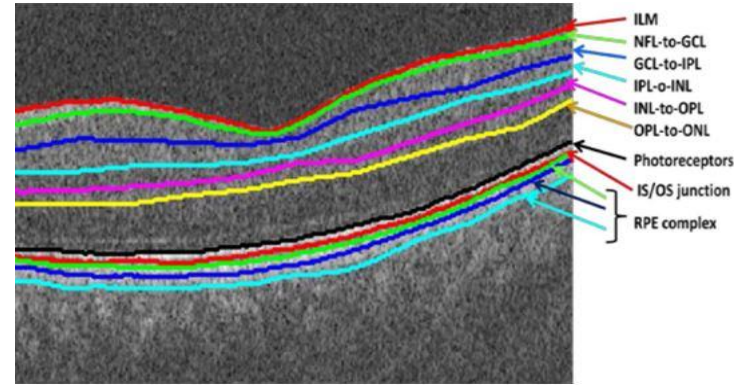

Figure 2: OCT Image showing different retinal layers (Image Courtesy: - [4])

Cheng et al. (2016) [4]: This paper proposes another strategy for speckle noise reduction in 3D OCT. The proposed technique models each A-scan as the aggregate of fundamental clean A-scan and noise. In the method, the neighbouring A-scans are aligned to the A-scan to be reconstructed and form a matrix together. Then low rank matrix completion using bilateral random projection is utilized to iteratively estimate the noise and recover the underlying clean $A$-scan [5]. Different from 2D scenarios where the clean image can be recovered from overlapping scan, here the method is to reconstruct each A-scan A(i,j) of a 3D scan from its neighbouring A-scans in the PXQ window centered at $(i, j)$. Here, the A-scans A(i,j) is formulated as a sum of its underlying clean part $c(i, j)$, a noise part $n(i, j)$, i.e.,

$$
A(i, j)=c(i, j)+n(i, j)
$$

This quantitatively demonstrates that the proposed speckle noise reduction technique makes the programmed retinal layer segmentation more exact, which is likewise an imperative viewpoint in assessing the technique which is shown in Figure 3-5.

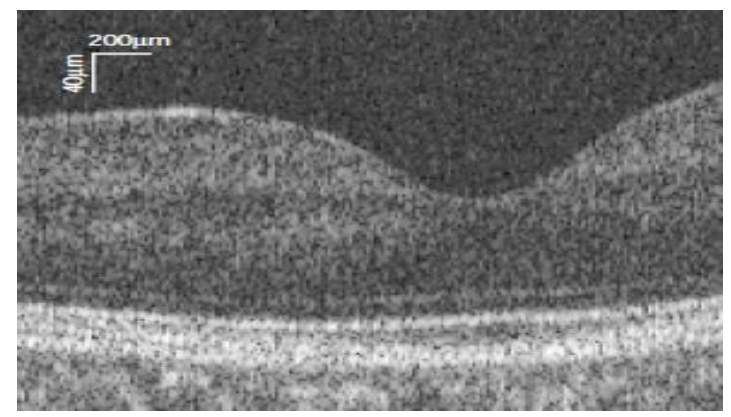

Figure 3: Region cropped from raw slice of a macula centered scan. (Image Courtesy: - [4])

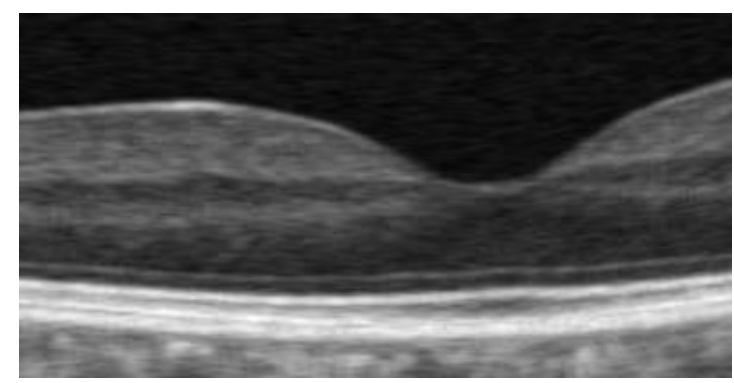

Figure 4: The results processed by the proposed method. (Image Courtesy: - [4])

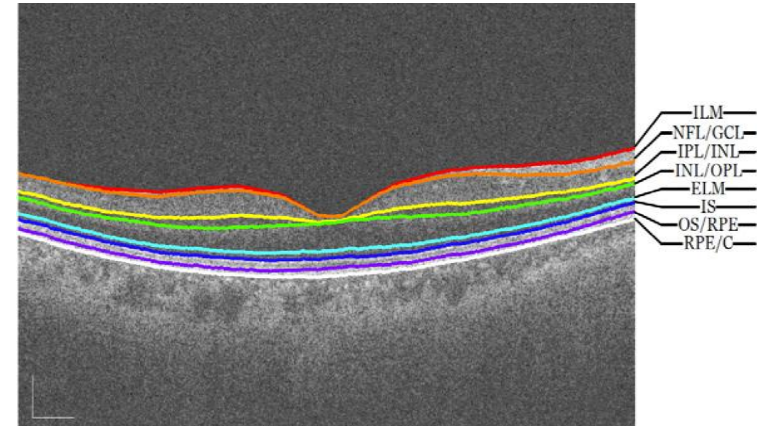

Figure 5: Illustration of layers: from top bottom, the eight boundaries correspond to boundary 1 to 8 . (Image Courtesy: - [4])

Anantrasirichai et al. (2013) [6]: Here the authors have proposed a novel strategy to remove speckle noise, while keeping valuable information contained in each retinal layer using Adaptive Bilateral Filter(AWBF). This helps in the keeping the texture property of OCT images intact. In traditional image filtering processes, a defined kernel is applied to each pixel and its neighbours of the target image. For smoothing the image, the low-pass filter is generally used to calculate a weighted average of pixel values in the neighbourhood.

The generally-used filter is a shift-invariant Gaussian filter, where both the closeness function $\mathrm{G}_{\sigma \mathrm{s}}(\bullet)$ and the similarity function $G_{\sigma r}(\bullet)$ are Gaussian functions with standard deviation $\sigma s$ and $\sigma r$, respectively. The equations involved in application of bilateral filters are given in Equation 2 and Equation 3. A new weighted average at pixel $\mathrm{p}$ with window space $\mathrm{S}$ can be written as in Equation 2,

Where $W p$ is for normalisation.

$\mathrm{BF}[\mathrm{Ip}]=\frac{1}{W p} \sum_{\mathrm{q} \in \mathrm{S}} G_{\sigma S}(|| \mathrm{p}-\mathrm{q}||) G_{\sigma r}(|\mathrm{p}-\mathrm{q}|) \mathrm{Iq}$

A general mathematical expression [7] for Adaptive Bilateral Filter $(\mathrm{ABF})$ is shown in Equation 3.

$\mathrm{ABF}[\mathrm{Ip}]=$

$\frac{1}{W p} \sum_{q \in S} w(p, q) G_{\sigma s}(|| \mathrm{p}-\mathrm{q}||) G_{\sigma r}(|\mathrm{p}-\mathrm{q}|) \mathrm{Iq}$

Where $w(p, q)$ is a local weight applied at pixel p related to neighbour q.

Outputs obtained after filtering using different values of standard deviation of closeness function $(\sigma s)$ and standard deviation of similarity function ( $\sigma r)$ are shown in Figure 6. Figure 7 shows the output comparison of raw images, despeckled images and enhanced images using AWBF.

Padmasini et. al (2014) [8]: In this paper the pre-processing method for de-noising the spectral domain OCT images is performed using Anisotropic Diffusion filtering [9]. The equation involved in calculating Shannon entropy is given in Equation 4

$S_{n}(\mu(x, y))=-\mu(x, y) \log _{2} \mu(x, y)(1-\mu(x, y)) \log _{2}(1-$ $\mu(x, y))$

Where $\mu(x, y)$ represent the degree of the pixel inten 


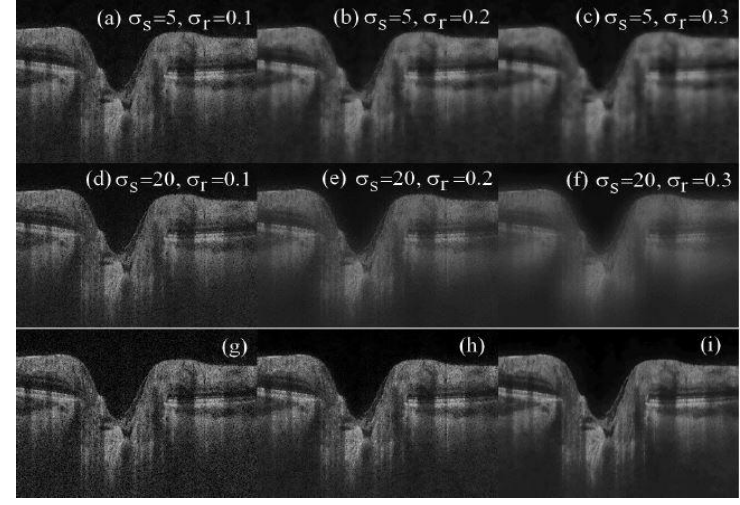

Figure 6: OCT image at the ONH. (a)-(f): smooth images with traditional bilateral filter with different parameters. (g) despeckled image. (h) smooth image with AWBF using single B-scan. (i) smooth image with AWBF using 3 Bscans. (Image Courtesy: - [6])

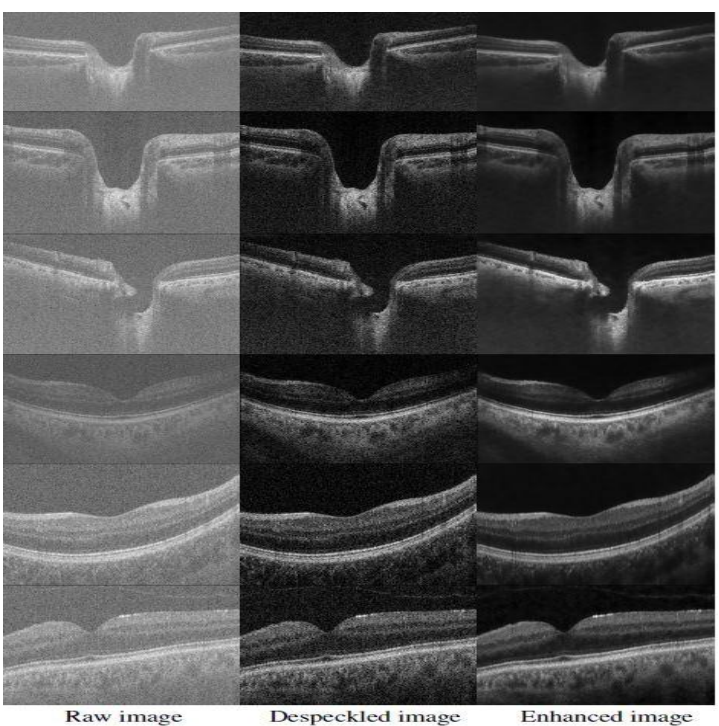

Figure 7: Enhanced OCT images of 6 eyes at the ONH (row 1-3) and the macula (row 4-6). (Image Courtesy: [6])

Where $\mu(x, y)$ represent the degree of the pixel intensities.

Anisotropic diffusion for images is given by partial differential equation (PDE) as shown below.

$\frac{\partial I}{\partial t}=\operatorname{div}(c(x, y, t) \nabla I)=\nabla c \cdot \nabla \mathrm{I}+\mathrm{c}(\mathrm{x}, \mathrm{y} \cdot \mathrm{t}) \Delta \mathrm{I}$

Where $\Delta$ and $\nabla$ are the Laplacian and gradient operators respectively, div is the divergence operator. The variable $c$ is the flux function. It controls the diffusion rate of the image. The locations of boundaries in the regions are absolute at the time $\mathrm{t}$, for the scale space variables. The divergence operator simplifies as:

$$
\operatorname{div}(c(x, y, t) \nabla I)=\frac{\partial}{\partial x}\left(c(x, y, t) I_{x}\right)
$$

Figure 8 shows the output of anisotropic diffusion filtered image.

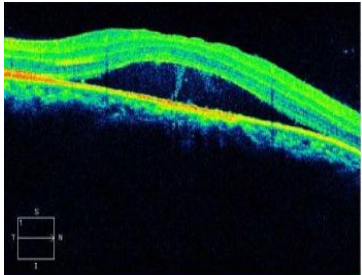

(a)

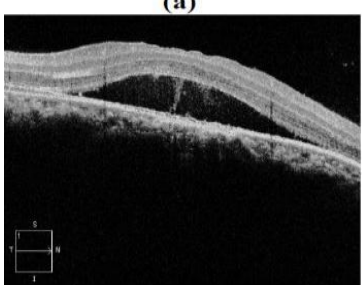

(c)

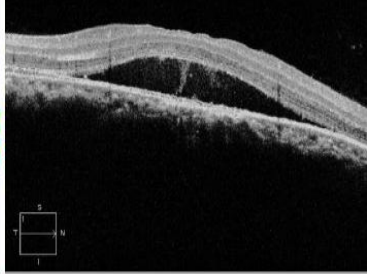

(b)

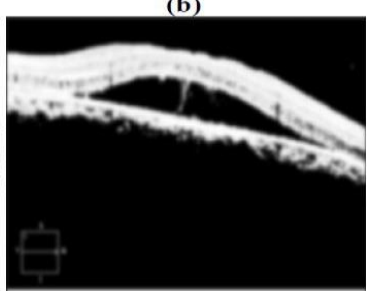

(d)
Figure 8: Output images a) Original image b) Normalized image c) Fuzzification output d) Anisotropic diffusion filter output. (Image Courtesy: - [8])

Abhishek et. al (2014) [10]: In this paper, an automated segmentation process to distinguish few intraretinal layers which are vital for edema recognition introduce in Spectral Domain Optical Coherence Tomography (SDOCT) images is shown. The layers segmented are Inner Limiting Layer (ILM) and Retinal Pigment Epithelium (RPE) layer and using thickness estimated between the ILM and RPE layer the image is categorised as edema or non-edema[11]. The graph based segmentation is exclusively in view of pixel intensity variation and distance between neighbouring pixels. Utilizing the weighing scheme and shortest path search, it facilitates the task by recognizing the neighbourhood pixel having same or comparable pixel intensity and connects it by a path having minimum weight having the minimum weight. The equation involved here is given in Equation 7.

$$
W_{a b}=2-\left(g_{a}+g_{b}\right)+W_{\min }
$$

Where $W_{a b}$ is the weight assigned to edges conne des $\mathrm{a} \& \mathrm{~b}$,

$g_{a}$ is the vertical gradient of the image at node a,

$g_{b}$ is the vertical gradient of the image at node $\mathrm{b}$,

$W_{\min }$ is the Minimum weight in the graph, a small positive number added for system stabilization.

The distance between ILM and RPE layers is shown in the form of a graph which is calculated using distance formula. The output images are shown in Figure 9 a) and b) and c)

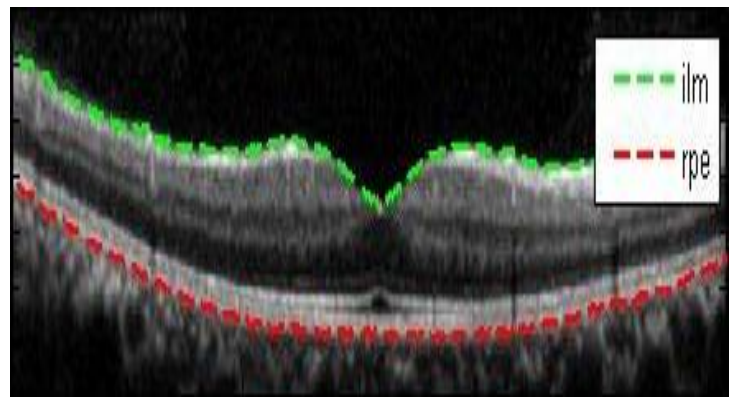

(a) 


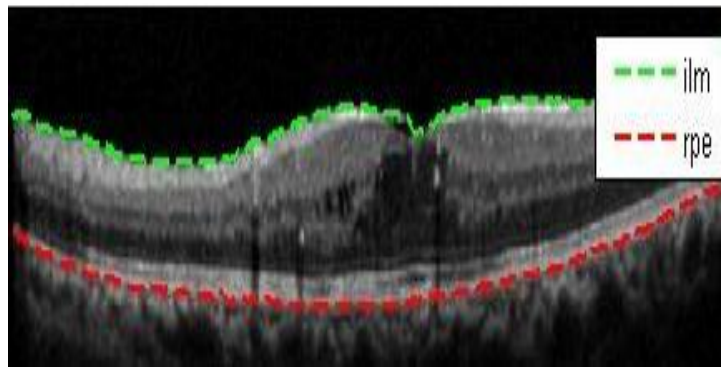

(b)

Figure 9: Output Images a) Segmented output (normal subject) b) Segmented output (DME subject). (Image Courtesy: - [10])

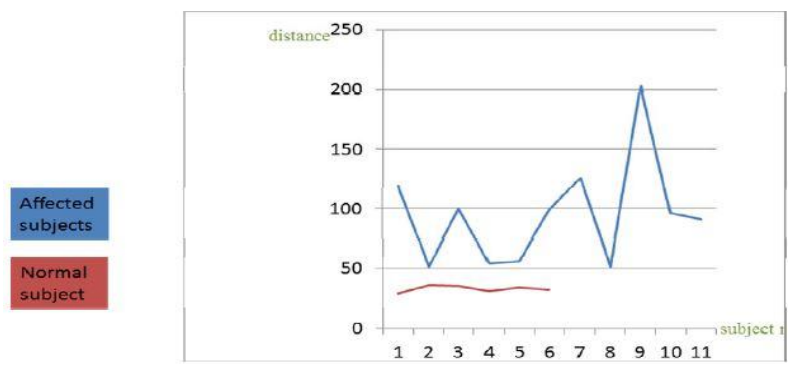

(c)

Fig. 9 c) Line graph for normal and DME affected subjects. (Image Courtesy: - [10])

Chiu et. al (2010) [12]: In this paper, the author presents an automatic approach for segmenting retinal layers in Spectral Domain OCT images using the graph theory algorithm and dynamic programming since manual segmentation of retinal images is a time consuming and subjective process. The paper discusses the general theory behind graph based segmentation as well as a generalized method for automatically segmenting layered structures. It also shows an implementation of the algorithm for segmenting eight retinal layer boundaries in normal adult eyes. The equation involved in the graph based segmentation is same as given in Equation 7. The block diagram for the layer segmentation process is shown in Figure 10.
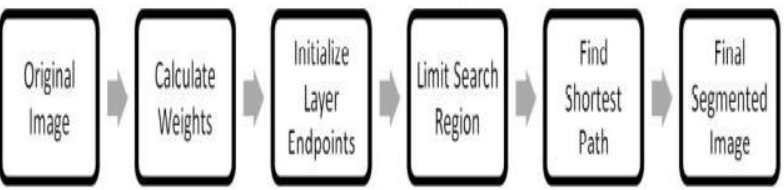

Figure 10: A generalized layer segmentation algorithm schematic. (Image Courtesy: - [12])

Segmentation of the OCT image is first done using Dijkstra's algorithm. This results in automatic endpoint initialization, and search space limitation. Then a connectivity based step where clusters associated with a particular layer are connected and modified. After this the search region limitation is applied to segment different layers [13]. Output obtained after segmenting the OCT image is shown in Figure 11 a) and the comparison output of automated verses manual segmentation is shown in Figure 11 b).

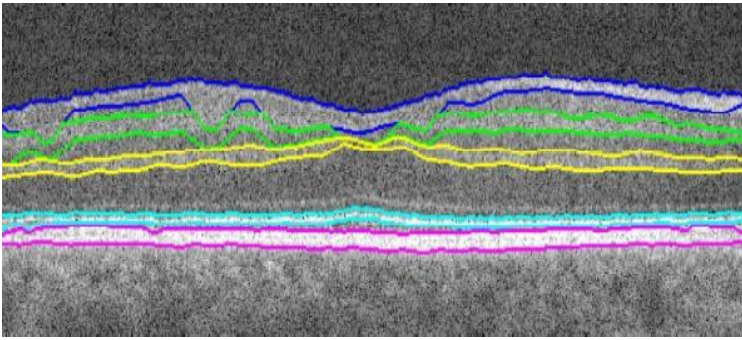

Figure 11 a): Connectivity-based segmentation of retinal layers. (Image Courtesy: - [12])

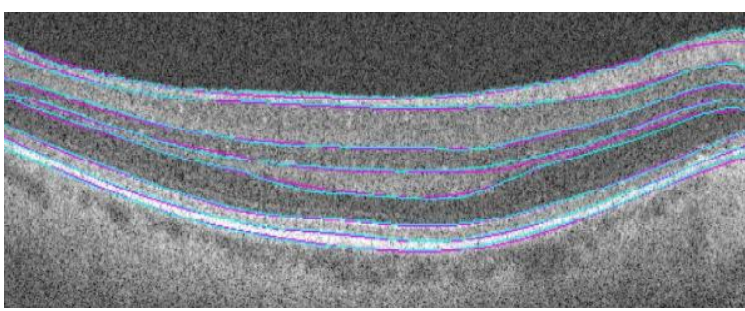

Figure 11 b) : Comparison of automatic (cyan) versus manual (magenta) segmentation. (Image Courtesy: - [12])

Lang et.al (2013) [14]: In this paper, the authors have created a random forest classifier which can segment eight retinal layers in macular cube images that is obtained by OCT. The random forest classifier is trained to learn about the boundary pixels between different retinal layers, produce an accurate probability map for each retinal boundary, which is then finally processed to finalise the boundaries [15]. After boundary refinement, the final segmented result is obtained using two techniques i.e. with a Canny (CAN) based boundary tracking algorithm, or an optimal graph search (GS) algorithm. The output obtained is shown in Figure 12.

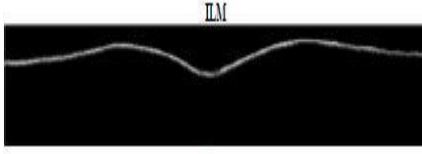

$\mathbb{P L}-\mathrm{NI}$

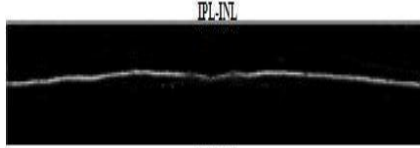

OPL-ONI

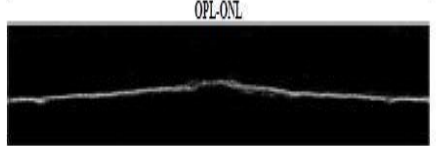

15.05
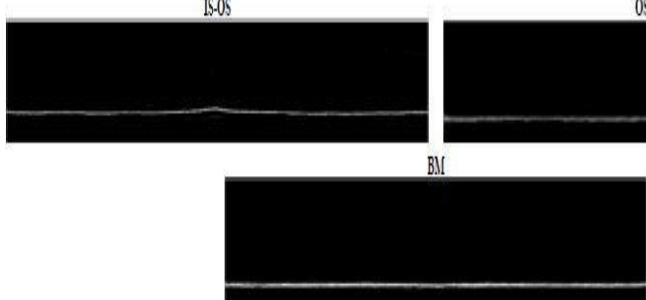

Figure 12: The probabilities for each boundary generated as the output of the random forest classifier. The probabilities are shown for each boundary, starting from the top of the retina to the bottom, going across each row. (Image Courtesy: - [14])

Duan et. al (2015) [16] In this paper the authors have proposed a method that is based on a coarse-to-fine approach to segmentation. It includes different stages. First, a 
variational retinex model that enhances the details and also corrects the intensity in-homogeneities present in the OCT images [17]. The retinex model is given as: $S=R L$

Where, $S$ is the observed image,

$R$ is the reflectance i.e. enhanced image with an interval $[0,1]$, $L$ is the illumination. To model the inverse problem additive version is obtained by taking logarithm to both sides of Equation 8 and is shown in Equation 9.

$$
s=r+l
$$

Now, for illumination $l$ to be smooth and reflectance $r$ to have sharp details i.e. edges, the variational function for image enhancement is given as in Equation 10.

$$
E(r)=\alpha \int_{\Omega} r^{2}+\int_{\Omega}|\nabla(s-r)|^{2}+\beta \int_{\Omega}\left|\nabla_{r}\right|
$$

Where $r$ is $\leq 0$ so that intensities of $R$ lies in $[0,1]$.

Second the layer boundaries are strengthened using anisotropic CED (Coherence Enhancing Diffusion) by using Equation 11.

$$
\partial_{t} u=\operatorname{div}\left(\boldsymbol{D}\left(\boldsymbol{J}_{p}\right) \nabla u\right)
$$

Where $\partial_{t} u$ denotes the first derivative with respect to diffusion time, and $\boldsymbol{J}_{p}$ is the smoothed version of the structure tensor $\boldsymbol{J}$ by using a Gaussian kernel with standard deviation $p$.

Third a nonlinear isotropic filter is used to smooth the processed OCT for the initial coarse segmentation as shown in Equation 12.

$$
u=\operatorname{div}\left(g\left(\left|\nabla u_{\sigma}\right|\right) \nabla u\right)
$$

Where $g\left(\left|\nabla u_{\sigma}\right|\right)$ is a non-increasing diffusivity, different from the structure tensor used in Equation 12. Function $g$ allows isotropic diffusion in flat regions and no diffusion near the edges.

Fourth, a high-pass un-sharp masking filter is applied that highlights the remaining fine and thin retinal layers and it results in the fine segmentation which is based on Equation 13.

$$
g=f+k\left(g-G_{\sigma} * f\right)
$$

where $f$ is blurred input image, $k$ is a positive parameter. Greater $k$ means high-boost emphasis of high frequencies (i.e. edges). $G_{\sigma}$ is Gaussian kernel with standard deviation $\sigma$. The output obtained is shown in Figure 13.
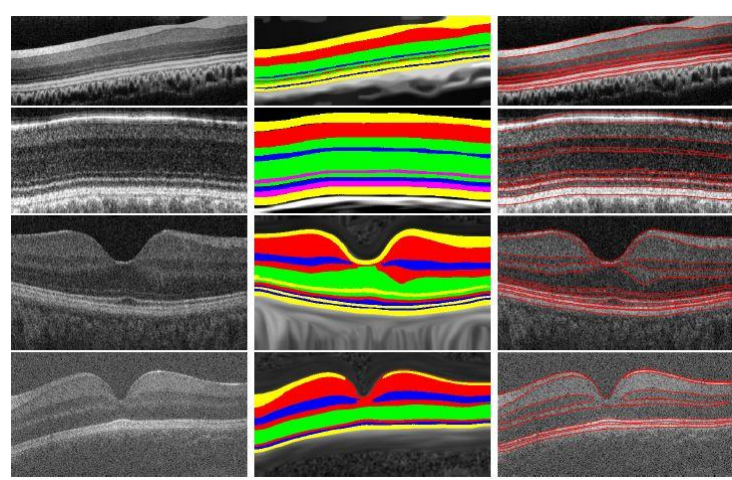

Figure 13: 1st column: Original images; 2nd column: Combined the two segmentation results with different colours drawn on different retinal layers; 3rd column:

Final results shown in reds. (Image Courtesy: - [16])

Amini et.al (2016) [18]: In this paper the authors suggested a method that is based on a nonlinear Gaussian transform which converts the probability distribution function (pdf) of each retinal layer into a Gaussian distribution [19]. The block diagram for the proposed method is given in Figure 14. In the first block (in the logarithmic domain) a suitable well-fitted mixture model and its parameters for the OCT image is found out. Here, the well-fitted model is referred to as a NormalLaplace mixture model. In the second block, each component of the mixture model is transformed to a Gaussian distribution having a specific mean and variance so that each enhanced component can be obtained. These components are then combined with each other in the third block to construct the entire enhanced image. Figure 15 shows the histogram and the best fitted Normal-Laplace mixture model for the sample of healthy OCT B-scan. Based on the results obtained Figure 16 shows the Chi Square measures which illustrates that the Normal-Laplace mixture model is better fitted to OCT data. Here, the proposed method was compared with the ContrastLimited Adaptive Histogram Equalization (CLAHE) method for checking its effectiveness in contrast enhancement as shown in Figure 17.

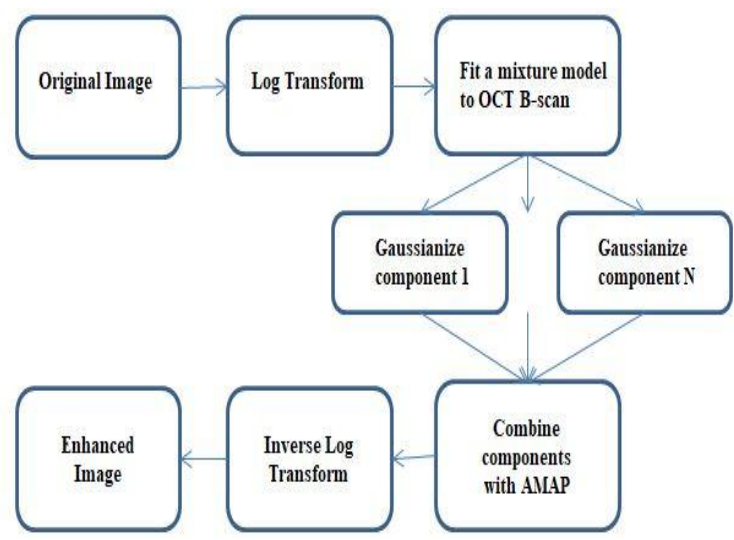

Figure 14: The block diagram of the proposed method. (Image Courtesy: - [18])

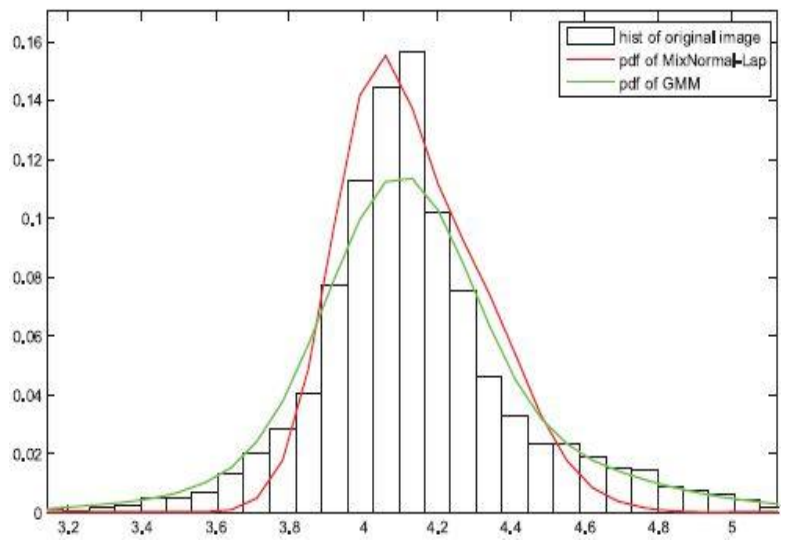

Figure 15: Comparison of the goodness of fit for Gaussian mixture model (GMM) and Normal-Laplace Mixture model to the histogram of a sample OCT. $X$-axis shows log-intensity values and $\mathrm{Y}$-axis indicates normalized number of pixels. (Image Courtesy: - [18]) 
๑.Gaussian dist $\quad$.Normal-Laplace dist

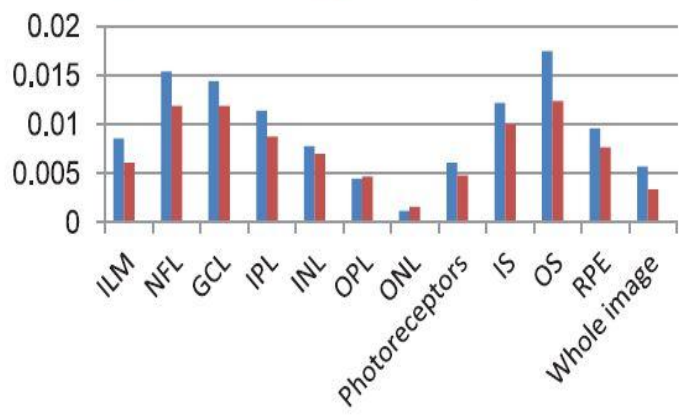

Figure 16: Comparison of Chi $\left(\chi^{2}\right)$ Square test results for Gaussian and Normal-Laplace distributions fitted on a sample OCT. (Image Courtesy: - [18])
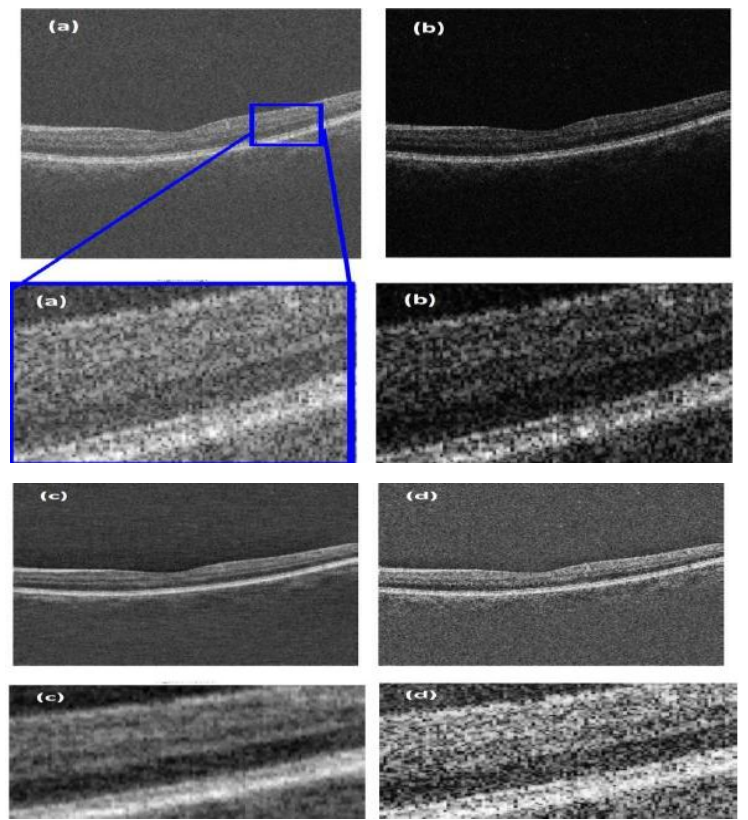

Figure 17: Contrast enhancement results in a sample Topcon image. (a) Original image, (b) proposed method, (c) transform histogram shaping method, (d) CLAHE method. The second row shows an enlargement of the specified region of first row images. (Image Courtesy: [18])

Roychowdhury et. al (2013) [20]: In this paper an automated process for localization of cysts in OCT images of patients having DME by estimating the area and location of the cystoid regions per image. These area estimates from adjacent OCT scans are then combined to obtain cystoid volumes. The images are segmented to detect six sub-retinal layers by iterative high-pass filtering. This step is then followed by the detection of the cystoid regions that may appear in the subretinal micro-structure. Here, the authors have extracted the sub-retinal layers by first estimating a mask of the region that contains the layer $(\mathrm{Gm})$, and then highpass filtering the image (Im) with the masked region of interest only shown in Equation 14. Masking the image helps to concentrate attention to a certain region of the image at a time, thus segmenting a complete region $\left(I_{R}\right)$ in one iteration.

$$
\begin{array}{r}
I_{R}=H P F(\operatorname{Im} \circ \mathrm{Gm}) \\
\Rightarrow I_{R}=I m \circ G m-\operatorname{LPF}(\operatorname{Im} \circ \mathrm{Gm})
\end{array}
$$

Here $H P F$ and $L P F$ represent the standard spatial high-pass filtered version and low-pass filtered version of the image, respectively, o represents per-element multiplication of matrices, or the Hadamard product. Cysts detection is done by using different threshold values. Here cyst is found by two algorithms based on cysts area estimation and cysts location estimation. Outputs obtained are shown in Figure 18 and Figure 19 below.

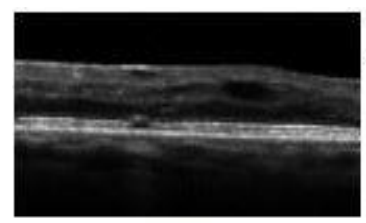

(a)

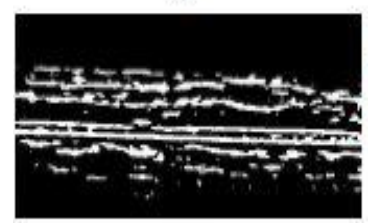

(c)

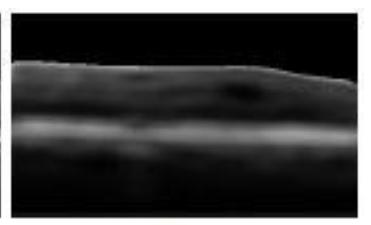

(b)

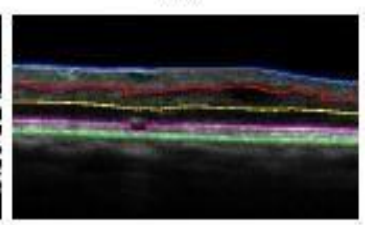

(d)
Figure 18: Automated segmentation of 6 sub-retinal layers. (a) Original Image. (b) LPF version of de-noised image. (c) HPF components detected after first iteration. (d) Final six layers colour overlaid on original image as: NFL (Blue), IPL/GL (Cyan), INL (Red), ONL (Yellow), PIS (Magenta), PE (Green). (Image Courtesy: - [20])

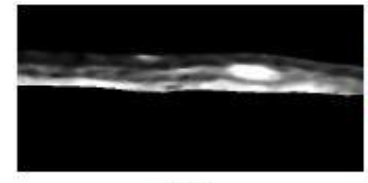

(a)

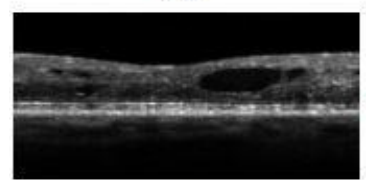

(c)

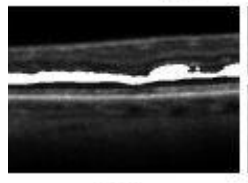

(e)

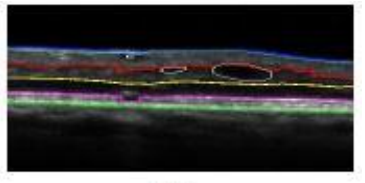

(b)

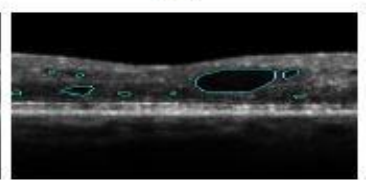

(d)

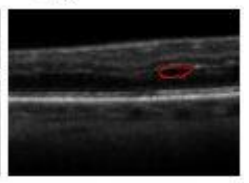

(g)
Figure 19: Cyst detection. (a) Candidate cystoid regions after contrast enhancement appear brighter than the immediate neighbourhood in the negative of the image within the NFL and PIS layers. (b) True cysts detected. (c) Actual image (d) True small cysts detected (e) Large cyst detected (f) Broken large cysts (g) True cysts detected after cyst decision algorithm is invoked. (Image Courtesy: - [20])

Hassan et. al (2016) [21] In this paper, the authors have proposed a fully automated method for the identification of Macular Edema (ME) from OCT images using Discriminant Analysis (DA) classifier. Here the classifier is trained by extracting three different features. These are maximum and minimum thickness between Inner Limiting Membrane (ILM) layer and choroid layer and the difference of maximum and minimum thickness. This involves the following processes: 
Pre-processing, feature extraction, discriminant analysis, and classification of ME based on the result obtained. Preprocessing involves resizing of image. In feature extraction part a three dimension feature vector $f t(x, y, z)$ is computed using thickness vector which contains three features that are the maximum thickness between ILM and choroid (f1), the minimum thickness between ILM and choroid (f2) and the difference between maximum and minimum thickness (f3) which is much less in case of healthy images as compared to ME case [22]. The equations involved are:

$$
f t(x, y, z)=[f 1, f 2, f 3]
$$

Where

$$
\begin{gathered}
f 1=\max \left(\left[I_{\text {choroid }}(x, y)-I_{I L M}(x, y) \mid\right)\right. \\
f 2=\min \left(\| I_{\text {choroid }}(x, y)-I_{I L M}(x, y) \mid\right) \\
f 3=f 1-f 2
\end{gathered}
$$

$I_{\text {Choroid }}(x, y)$ is the choroid layer pixel in each A-Scan of $I_{\text {Binary }}(x, y)$ and $I_{I L M}(x, y)$ is the ILM layer pixel in each AScan of $I_{\text {Binary }}(x, y)$. After this the OCT images are applied to the Discriminant Analyser and the images are classified as ME positive or healthy. The output obtained is shown in Figure 20.

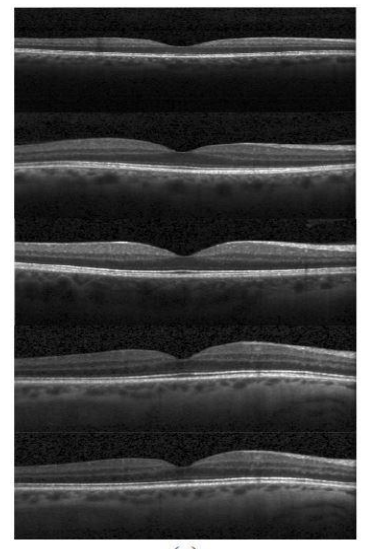

(a)

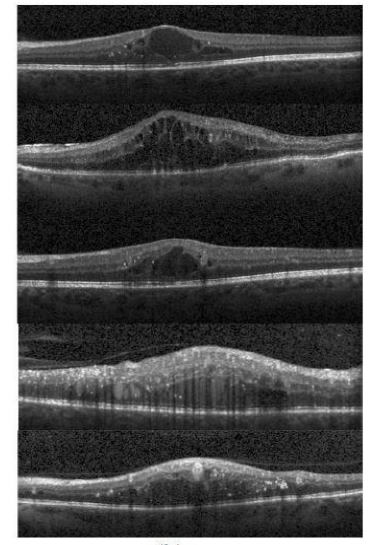

(b)
Figure 20 (a) Classified as 'Healthy' (b) Classified as ME Positive. (Image Courtesy: - [21])

The summary of all the works discussed in this review section are given in Table 1. This gives instant information about objective of the work done, method followed, image database used and the results obtained in each case.

\section{CONCLUSION}

The literature survey shows that OCT images can be used to analyse a variety of retinal diseases. Different type of noises present in OCT image and the effective filter to remove the noise are also discussed here. Many research works on segmentation of various retinal layers are presented. The data obtained from thickness measurement and texture measurement of the retinal layers, different retinal disorders can be detected. Current research in the segmentation of OCT images is striving towards improving the accuracy, precision, and computational speed of segmentation methods, as well as reducing the amount of manual interaction. Segmentation algorithms have the capability to run in parallel with the OCT scanning method and to provide a concrete support for clinical decision making in real time. Since OCT allows real-time data acquisition, future research will strive towards improving automation and data evaluation in to support retinal disease screening and diagnosis in a more improved way.

\section{REFERENCES}

[1] R. D. Chaudhari, A.A. Pawar and R.S. Deore, 2013, "The Historical Development Of Biometric Authentication Techniques: A Recent Overview", International Journal of Engineering Research and Technology (IJERT), Vol.2, Issue 10.

[2] D. Huang, E. A. Swanson, C. P. Lin, J. S. Schuman, W. G. Stinson, W. Chang, M. R. Hee, T. Flotte, K. Gregory, C. A. Puliafito, and J. G. Fujimoto, , 1991, "Optical coherence tomography," Science, vol. 254, no. 5035, pp. $1178-1181$.

[3] G.D. Hildebrand and A.R. Fielder, 2011, "Anatomy and Physiology of the Retina", Springer-Verlag Berlin Heidelberg.

[4] J. Cheng, D. Tao, Y. Quan, D. W. K. Wong, Member, G.C. M. Cheung, M. Akiba and J. Liu, 2016, "Speckle Reduction in 3D Optical Coherence Tomography of Retina by A-Scan Reconstruction", IEEE Transactions on Medical Imaging.

[5] A. Ozcan, A. Bilenca, A. E. Desjardins, B. E. Bouma, and G. J. Tearney, 2007, "Speckle reduction in optical coherence tomography images using digital filtering," J. Opt. Soc. of Am. A, vol. 24(7), pp. 1901-1910.

[6] N. Anantrasirichai, L. Nicholson, J. E. Morgan, I.Erchovay, A. Achim, 2013 "Adaptive-Weighted Bilateral Filtering For Optical Coherence Tomography”,978-1-4799-2341-0/13/2013 IEEE, ICIP.

[7] B. Zhang and J.P. Allebach, 2007 ,"Adaptive bilateral filter for sharpness enhancement and noise removal," in Image Processing, ICIP 2007. IEEE International Conference on, Oct. 2007, vol. 4, pp. IV -417 -IV - 420.

[8] N.Padmasini, K.S.Abbirame, R. Umamaheswari and S.Y. Mohamed, 2014 "Speckle Noise Reduction in Spectral Domain Optical Coherence Tomography Retinal Images Using Anisotropic Diffusion Filtering”, International Conference on Science, Engineering and Management Research (ICSEMR 2014).

[9] Yingtao Zhang, H.D.Cheng, JiaweiTian and JianhuaHuang a, XianglongTang, 2010, "Fractional subpixel diffusion and fuzzy logic approach for ultrasound speckle reduction", in Elsevier journal of pattern recognition, Vol. 43, 2962-2970.

[10] A. M. Abhishek, T. T. J. M. Berendschot, S. V. Rao, and S. Dabir, 2014, "Segmentation and Analysis of Retinal Layers (ILM \& RPE) in Optical Coherence Tomography Images with Edema", IEEE Conference on Biomedical Engineering and Sciences, Miri, Sarawak, Malaysia.

[11] Raheleh Kafieh, Hossein Rabbani, Saeed Kermani, 2013, "A Review of Algorithms for Segmentation of Optical Coherence Tomography from Retina" Journal of Medical Signals \& Sensors.

[12] S. J. Chiu, X. T. Li, P. Nicholas, C. A. Toth, J. A. Izatt and S. Farsiu, 2010, "Automatic segmentation of seven retinal layers in SDOCT images congruent with expert manual segmentation", Vol. 18, No. 18 / OPTICS EXPRESS 19413. 
[13] A. Mishra, A. Wong, K. Bizheva, and D. A. Clausi, 2009, "Intra-retinal layer segmentation in optical coherence tomography images," Opt. Express 17(26), 23719-23728.

[14] A. Lang, A. Carass,M. Hauser, E. S. Sotirchos, P. A. Calabresi, H. S. Ying, and J.L. Prince, 2013, "Retinal layer segmentation of macular OCT images using boundary Classification", Vol. 4, No. 7 | DOI:10.1364/BOE.4.001133 | BIOMEDICAL OPTICS EXPRESS 1133.

[15] A. Lang, A. Carass, E. Sotirchos, P. Calabresi, and J. L. Prince, 2013, "Segmentation of retinal OCT images using a random forest classifier," Proc. SPIE 8669, 86690R.

[16] J. Duan, C. Tenchy, I. Gottlobz, F. Proudlockz, L. Bai, 2015, "Optical Coherence Tomography Image Segmentation”, 978-1-4799-8339-1/15 IEEE, ICIP, 2015.

[17] Ron Kimmel, Michael Elad, Doron Shaked, Renato Keshet, and Irwin Sobel, 2003,"A variational framework for retinex," International Journal of computer vision, vol. 52 , no. 1 .

[18] Z.Amini and H. Rabbani, 2016, "Statistical Modeling of Retinal Optical Coherence Tomography", IEEE
TRANSACTIONS ON MEDICAL IMAGING, VOL. 35, NO. 6

[19] D. Erdogmus, R. Jenssen, Y. N. Rao, and J. C. Principe, 2006, "Gaussianization: An efficient multivariate density estimation technique for statistical signal processing," J. VLSI Signal Process. Syst. Signal, Image Video Technol., vol. 45 , pp. 67-83.

[20] S. Roychowdhury, D. D. Koozekanani, S. Radwan and K. K. Parhi, 2013,“Automated Localization of Cysts in Diabetic Macular Edema using Optical Coherence Tomography Images", 978-1-4577-0216-7/13, 35th Annual International Conference of the IEEE EMBS Osaka, Japan

[21] B. Hassan and G. Raja, 2016, "Fully Automated Assessment of Macular Edema using Optical Coherence Tomography (OCT) Images", 978-1-4673-87538/16/2016 IEEE.

[22] L. Zhang, W. Zhu, F. Shi, H. Chen, X. Chen, 2015, "Automated Segmentation Of Intraretinal Cystoid Macular Edema For Retinal 3D OCT Images With Macular Hole" International Symposium on Biomedical Imaging, vol. 12, pp. $1494-1497$.

Table 1. Summary of the literature review:

\begin{tabular}{|c|c|c|c|c|c|}
\hline Year & Authors & Research Work & Images Used & Methods & Parameters and Results \\
\hline 2016 & Cheng et al. & $\begin{array}{l}\text { Speckle Noise } \\
\text { Reduction }\end{array}$ & $\begin{array}{l}20 \text { images using the } \\
\text { ATLANTIS 3D-256 mode. }\end{array}$ & $\begin{array}{l}\text { A-scan Registration and } \\
\text { A-scan Reconstruction. }\end{array}$ & $\begin{array}{l}\text { MSE, PSNR and } \\
\text { MSSIM are calculated } \\
\text { and compared. }\end{array}$ \\
\hline 2013 & $\begin{array}{l}\text { Anantrasirichai } \\
\text { et al. }\end{array}$ & $\begin{array}{l}\text { Speckle Noise } \\
\text { Reduction }\end{array}$ & $\begin{array}{l}24 \text { retinal OCT images, of } \\
\text { which } 14 \text { normal eyes and } \\
10 \text { glaucoma eyes. }\end{array}$ & $\begin{array}{l}\text { Wavelet-based de- } \\
\text { speckling, wavelet based } \\
\text { image registration and } \\
\text { adaptive-weighted } \\
\text { bilateral filtering. }\end{array}$ & $\begin{array}{l}\text { Run-length } \\
\text { measurement, DTCWT, } \\
\text { Grey-level co- } \\
\text { occurrence matrix } \\
(\text { GLCM), Local Binary } \\
\text { Pattern (LBP) and } \\
\text { Granulometry. }\end{array}$ \\
\hline 2014 & Padmasini et. al & $\begin{array}{l}\text { Speckle Noise } \\
\text { Reduction }\end{array}$ & $\begin{array}{l}30 \text { images using Cirrus } \\
\text { HD-OCT, of which } 2 \\
\text { normal eyes and } 28 \\
\text { abnormal eyes }\end{array}$ & $\begin{array}{l}\text { Anisotropic diffusion } \\
\text { filtering using fuzzy } \\
\text { logic approach. }\end{array}$ & $\begin{array}{lll}\text { PSNR, SNR, CNR, } \\
\text { SSIM. }\end{array}$ \\
\hline 2014 & Abhishek et. al & Segmentation & $\begin{array}{l}21 \text { images, of which } 9 \\
\text { normal and } 12 \text { abnormal }\end{array}$ & $\begin{array}{l}\text { Canny edge, graph } \\
\text { theory }\end{array}$ & $\begin{array}{lll}2 \text { layers } & \text { (ILM, RPE) } \\
\text { detected with } & 85.7 \% \\
\text { accuracy. } & & \\
\end{array}$ \\
\hline 2010 & Chiu et. al & Segmentation & $\begin{array}{l}\text { Volumetric scans } \text { were } \\
\text { acquired from } 10 \text { normal } \\
\text { adult subjects in an SDOCT } \\
\text { imaging systems. }\end{array}$ & Graph cut method & $\begin{array}{l}8 \text { layers detected, } \\
\text { normalized mean and } \\
\text { standard deviation. }\end{array}$ \\
\hline 2013 & Lang et.al & Segmentation & $\begin{array}{l}35 \text { Images using a Spectralis } \\
\text { OCT system of which } 14 \\
\text { are healthy and } 21 \text { diseased. }\end{array}$ & $\begin{array}{l}\text { Random Forest } \\
\text { classifier followed by } \\
\text { canny edge detection or } \\
\text { graph search. }\end{array}$ & $\begin{array}{l}8 \text { retinal layers, absolute } \\
\text { error of retinal thickness } \\
\text { measurement. }\end{array}$ \\
\hline 2015 & Duan et. al & Segmentation & Not specified. & $\begin{array}{lr}\text { Variational } & \text { Retinax } \\
\text { Model, } & \text { Anisotropic } \\
\text { diffusion, } & \text { Nonlinear } \\
\text { diffusion, } & \text { Isotropic } \\
\text { diffusion, } & \text { Unsharp } \\
\text { masking } & \end{array}$ & $\begin{array}{l}7 \quad \text { retinal layers, } \\
\text { removed speckle noise, } \\
\text { connected } \\
\text { discontinuous } \\
\begin{array}{l}\text { boundaries, as ayer } \\
\text { enhances/ well as }\end{array} \\
\text { sharpens }\end{array}$ \\
\hline
\end{tabular}


International Journal of Computer Applications (0975 - 8887)

Volume 182 - No. 17, September 2018

\begin{tabular}{|c|c|c|c|c|c|}
\hline & & & & & weak image features. \\
\hline 2016 & Amini et.al & Segmentation & $\begin{array}{l}130 \text { B-scans from the } \\
\text { Topcon dataset and } 60 \text { B- } \\
\text { scans from } \\
\text { Zeiss. }\end{array}$ & $\begin{array}{l}\text { Statistical modelling } \\
\text { known as } \\
\text { Gaussianization by } \\
\text { calculating pdf of } \\
\text { different retinal layers. }\end{array}$ & $\begin{array}{lr}\text { Improved } & \text { contrast } \\
\text { enhancement } & \text { and } \\
\text { accurate segmentation } \\
\text { of intra-retinal layers. }\end{array}$ \\
\hline 2013 & $\begin{array}{l}\text { Roychowdhury } \\
\text { et. al }\end{array}$ & Cysts Detection & $\begin{array}{l}120 \text { images from } 25 \text { diabetic } \\
\text { patients with DME using the } \\
\text { Heidelberg Spectralis } \\
\text { imaging systems. }\end{array}$ & $\begin{array}{l}\text { High pass filtering and } \\
\text { masking followed by } \\
\text { cyst area and } \\
\text { localization estimation }\end{array}$ & $\begin{array}{l}90 \% \text { correlation } \\
\text { between the estimated } \\
\text { cystoid area and the } \\
\text { manually marked area, } \\
\text { and a mean error of } \\
4.6 \% \text {. Cysts detected in } \\
\text { the IPL region, INR and } \\
\text { ONR with an accuracy } \\
\text { of } 88 \%, 86 \% \text { and } 80 \% \text {, } \\
\text { respectively. }\end{array}$ \\
\hline 2016 & Hassan et. al & Macular Edema & $\begin{array}{l}30 \text { SDOCT images of which } \\
15 \text { healthy and } 15 \text { ME } \\
\text { effected. }\end{array}$ & Discriminant Analysis & $\begin{array}{l}100 \% \text { of } \mathrm{ME} \text { patients } \\
\text { and } 93.33 \% \text { of healthy } \\
\text { patients. }\end{array}$ \\
\hline
\end{tabular}

